\title{
Programmed Synthesis of Tetraarylthieno[3,2-b]thiophene by Site-Selective Suzuki Cross-Coupling Reactions of Tetrabromothieno[3,2-b]thiophene
}

\author{
Hien Nguyen, ${ }^{* a}$ Dung Xuan Nguyen, ${ }^{\mathrm{a}}$ Thinh Quang Tran, ${ }^{\mathrm{a}}$ Binh Ngoc Vo, ${ }^{\mathrm{a}}$ Thao Huong Nguyen, ${ }^{\mathrm{a}}$ Thi Minh \\ Ha Vuong, ${ }^{\text {, Tung T. Dang*c,1 }}$ \\ ${ }^{a}$ Department of Chemistry, Hanoi National University of Education, 136 Xuanthuy Street, Caugiay District, Hanoi, Vietnam \\ E-mail: hiennguyenp@yahoo.com \\ ${ }^{\text {b }}$ Laboratoire de Chimie Moléculaire et Thio-organique, 6 Boulevard Maréchal Juin, 14050 Caen, France \\ c Center d'elaboration de matériaux et d'etudes structurales, 29 rue Jeanne Marvig BP 94347, 31055 Toulouse Cedex 4, France \\ E-mail: dang.thanhtung@gmail.com
}

Received: 08.07.2013; Accepted after revision: 02.10.2013

\begin{abstract}
Thieno[3,2-b]thiophene is a structural motif that can be found in many important organic materials. A number of mono-, diand tetraarylthieno[3,2-b]thiophenes are reported herein.

Key words: cross-coupling reactions, palladium, Suzuki-Miyaura reaction, site selectivity, thieno[3,2-b]thiophene
\end{abstract}

Thieno[3,2-b]thiophene ${ }^{2}$ is a structural motif present in a wide range of conducting polymers, $p$-type organic semiconductors, optoelectronics, nonlinear optics and electroluminescence materials. Thieno[3,2-b]thiophene can be used as a starting material in the synthesis of oligo-functionalized thieno[3,2-b]thiophenes, thienoacenes and helical thienoacenes, which are conducting polymers and chromophores. ${ }^{2 b, 3}$ In 2006, McCulloch et al. reported a liquid-crystalline semiconducting polymer (PBTTT) containing thieno[3,2- $b]$ thiophene moieties with a very high charge-carrier mobility (Figure 1). ${ }^{4 a}$ Recently, dinaphtho[2,3-b:2', $\left.3^{\prime}-f\right]$ thieno[3,2- $\left.b\right]$ thiophene (DNTT) and alkylated benzothieno[3,2-b][1]benzothiophene $\left(\mathrm{C}_{13} \mathrm{BTBT}\right)$ were shown to demonstrate a very high thin film mobility of $3.1 \mathrm{~cm}^{2} / \mathrm{Vs}$ and $17.2 \mathrm{~cm}^{2} / \mathrm{Vs}$, respectively, in VD-OFETs. ${ }^{4 b, c}$ Due to intermolecular sulfur-sulfur interactions, materials containing thieno[3,2-b]thiophene may increase the electronic transport between neighboring molecules. The introduction of substituents into the core structure of materials may change electronic properties, solubility as well as molecular packing. ${ }^{2 \mathrm{~m}}$ For tuning electronic properties, heterocycles have been widely functionalized by many methods, especially, by palladium(0)catalyzed cross-coupling reactions. ${ }^{5}$ It was previously shown that polyhalogenated heterocycles can be regioselectively functionalized by palladium-catalyzed crosscoupling reactions at the carbon-halogen bonds adjacent to the heteroatom. ${ }^{5}$ These were controlled by both electronic and steric factors. We recently reported the methodologies for functionalization of $N$-methyltetrabromopyrrole, ${ }^{6}$ tetrabromothiophene, ${ }^{6}$ tetrabromoselenophene ${ }^{7}$

SYNLETT 2014, 25, 0093-0096

Advanced online publication: 04.12.2013

DOI: 10.1055/s-0033-1340481; Art ID: ST-2013-W0610-L

C Georg Thieme Verlag Stuttgart · New York

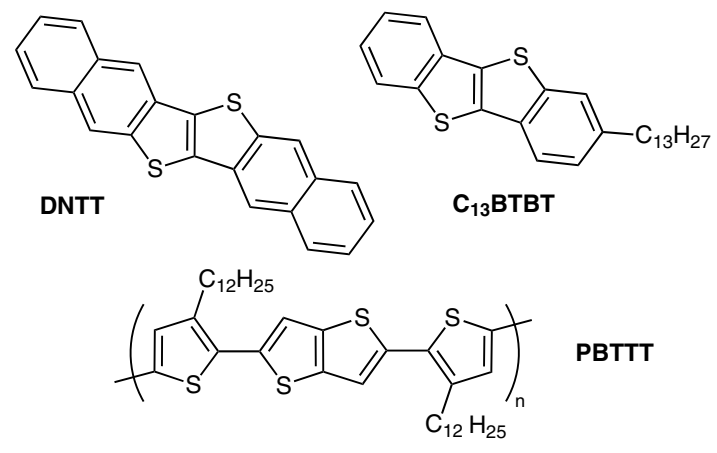

Figure 1 Some organic materials containing thieno[3,2-b]thiophene

and tetrabromofuran, ${ }^{8}$ based on site-selective palladium(0)-catalyzed Suzuki reactions.

Due to the importance of thieno[3,2-b]thiophene in materials science, we were interested in developing a sequential process for the functionalization of thieno[3,2$b]$ thiophene via site-selective palladium(0)-catalyzed Suzuki reactions of tetrabromothieno[3,2-b]thiophene with boronic acids. We report herein an efficient synthesis of mono-, di- and tetraarylthieno[3,2-b]thiophene using this strategy.

The Suzuki-Miyaura reactions of $\mathbf{1}^{9}$ (1.0 equiv) with a series of boronic acids (1.2 equiv) resulted in a site-selective formation of 2-aryl-3,5,6-tribromothieno[3,2-b] thiophenes $\mathbf{2 a}-\mathbf{j}^{5}$ in $25-80 \%$ yields (Scheme 1 and Table 1). The conditions used were optimized with regard to temperature, solvent, base additive, and water additive. $\mathrm{Pd}\left(\mathrm{PPh}_{3}\right)_{4}$ was found to be an efficient catalyst for the current reaction. Other well-known catalyst systems, such as $\mathrm{Pd}(\mathrm{OAc})_{2} / \mathrm{X}-\mathrm{Phos}$, resulted in lower yields of the desired products. All reactions were carried out at $90-110{ }^{\circ} \mathrm{C}$ in 4-6 hours.
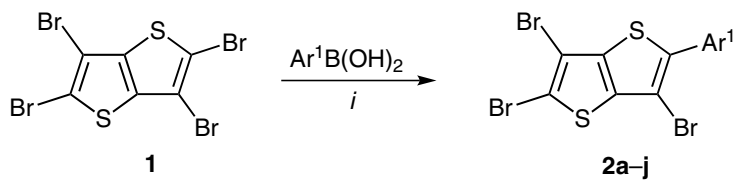

Scheme 1 Synthesis of $\mathbf{2 a}-\mathbf{j}$. Reagents and conditions: (i) $\mathbf{1}$ (1.0 equiv), $\mathrm{Ar}^{1} \mathrm{~B}(\mathrm{OH})_{2}$ (1.2 equiv), $\mathrm{Pd}\left(\mathrm{PPh}_{3}\right)_{4}(5 \mathrm{~mol} \%), \mathrm{K}_{3} \mathrm{PO}_{4}(2.0$ equiv), $110^{\circ} \mathrm{C}, 4-6 \mathrm{~h}$, solvent (see Table 1 ). 
Table 1 Synthesis of 2-Aryl-3,5,6-tribromothieno[3,2-b]thiophene $\mathbf{2 a - j}$

\begin{tabular}{llll}
\hline 2 & $\mathrm{Ar}^{1} \mathrm{~B}(\mathrm{OH})_{2}$ used & $\begin{array}{l}\text { Solvent } / \mathrm{H}_{2} \mathrm{O} \\
(4: 1)\end{array}$ & $\begin{array}{l}\mathrm{Yield}^{\mathrm{a}} \\
(\%)\end{array}$ \\
\hline a & $\mathrm{PhB}(\mathrm{OH})_{2}$ & toluene & 51 \\
b & $4-\mathrm{MeC}_{6} \mathrm{H}_{4} \mathrm{~B}(\mathrm{OH})_{2}$ & toluene & 50 \\
c & $3,5-\mathrm{Me}_{2} \mathrm{C}_{6} \mathrm{H}_{3} \mathrm{~B}(\mathrm{OH})_{2}$ & toluene & 80 \\
d & $4-t-\mathrm{BuC}_{6} \mathrm{H}_{4} \mathrm{~B}(\mathrm{OH})_{2}$ & toluene & 55 \\
e & $4-\mathrm{MeOC}_{6} \mathrm{H}_{4} \mathrm{~B}(\mathrm{OH})_{2}$ & $1,4-$ dioxane & 25 \\
f & $3-\mathrm{NaphthylB}(\mathrm{OH})_{2}$ & toluene & 42 \\
g & $3-\mathrm{EtOC}{ }_{6} \mathrm{H}_{4} \mathrm{~B}(\mathrm{OH})_{2}$ & toluene & 55 \\
h & $4-\mathrm{F}_{3} \mathrm{CC}_{6} \mathrm{H}_{4} \mathrm{~B}(\mathrm{OH})_{2}$ & toluene & 68 \\
i & acenaphthene-5-boronic acid & toluene & 52 \\
j & $3-\mathrm{Me}-4-\mathrm{MeOC}_{6} \mathrm{H}_{3} \mathrm{~B}(\mathrm{OH})_{2}$ & toluene & 50 \\
\hline
\end{tabular}

${ }^{a}$ Isolated yields.

${ }^{\mathrm{b}}$ See refs. $7 \mathrm{~b}$ and 11 .

The structures of the coupling products were established by spectroscopic methods. To confirm the site-selectivity of the Suzuki-Miyaura reactions, the structure of $\mathbf{2 d}$ was clearly characterized by X-ray crystal structure and ${ }^{1} \mathrm{H}$ NMR and ${ }^{13} \mathrm{C}$ NMR analysis (see Figure 2). In agreement with previous reports, ${ }^{7,8,10}$ the Suzuki reaction proceeded regioselectively due to the preference of the multibrominated heterocycles to undergo oxidative addition with $\operatorname{Pd}(0)$ at the most electron-deficient carbon atoms, for example $\mathrm{C} 2$ and/or C5 in the case of 2,3,5,6-tetrabromothieno[3,2-b] thiophene. ${ }^{7 \mathrm{a}-\mathrm{c}}$

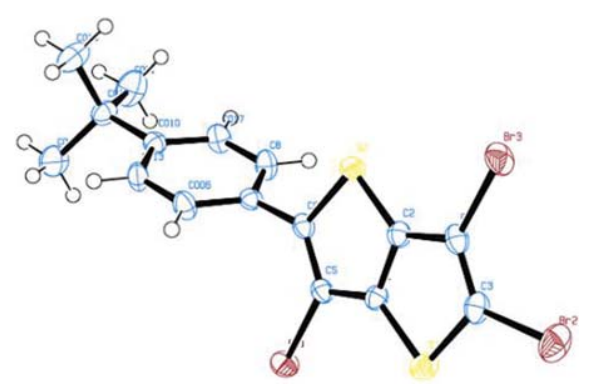

Figure 2 X-ray crystal structure of $\mathbf{2 d}$

The Suzuki-Miyaura reactions of 1 (1.0 equiv) with 2.2 equivalents of arylboronic acids afforded symmetrical 2,5-diaryl-3,6-dibromothieno[3,2-b]thiophenes $\mathbf{3 a}-\mathbf{d}^{11}$ in $30-70 \%$ yield (Scheme 2 and Table 2). The reactions again proceeded with very good site-selectivity as confirmed by ${ }^{1} \mathrm{H}$ NMR and ${ }^{13} \mathrm{C}$ NMR.

Similarly, the unsymmetrically disubstituted 2,5-diaryl3,6-dibromothieno[3,2-b]thiophenes $\mathbf{4 a}-\mathbf{c}$ could also be

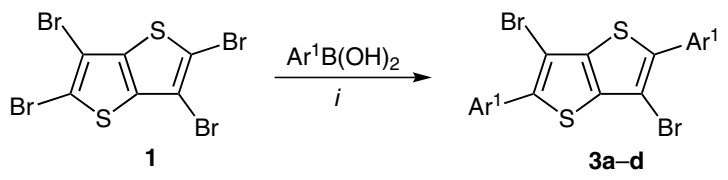

Scheme 2 Synthesis of 3a-d. Reagents and conditions: (i) 1 (1.0 equiv), $\mathrm{Ar}^{1} \mathrm{~B}(\mathrm{OH})_{2}$ (2.2 equiv), $\left.\mathrm{Pd}_{(} \mathrm{Ph}_{3} \mathrm{P}\right)_{4}(10 \mathrm{~mol} \%), \mathrm{K}_{3} \mathrm{PO}_{4}(4.0$ equiv), toluene- $\mathrm{H}_{2} \mathrm{O}(4: 1), 110{ }^{\circ} \mathrm{C}, 4-6 \mathrm{~h}$.

Table 2 Synthesis of Symmetrical 2,5-Diaryl-3,6-dibromothieno[3,2- $b]$ thiophenes 3a-d

\begin{tabular}{lll}
\hline 3 & $\mathrm{Ar}^{1} \mathrm{~B}(\mathrm{OH})_{2}$ & Yield $^{\mathrm{a}}(\%)$ \\
\hline a & $\mathrm{PhB}(\mathrm{OH})_{2}$ & 58 \\
b & $4-\mathrm{MeC}_{6} \mathrm{H}_{4} \mathrm{~B}(\mathrm{OH})_{2}$ & 70 \\
c & $3,5-\mathrm{Me}_{2} \mathrm{C}_{6} \mathrm{H}_{3} \mathrm{~B}(\mathrm{OH})_{2}$ & 42 \\
d & $4-t-\mathrm{BuC}_{6} \mathrm{H}_{4} \mathrm{~B}(\mathrm{OH})_{2}$ & 30 \\
\hline
\end{tabular}

${ }^{\mathrm{a}}$ Isolated yields.

synthesized by our method with predictable selectivity (Scheme 3 and Table 3). ${ }^{12}$
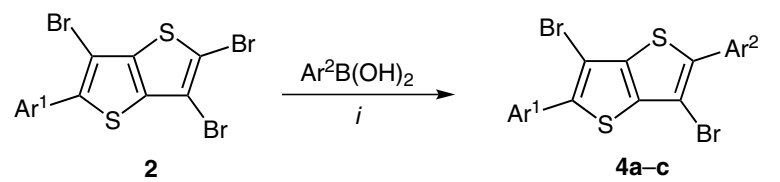

Scheme 3 Synthesis of $\mathbf{4 a - c . ~ R e a g e n t s ~ a n d ~ c o n d i t i o n s : ~ ( i ) ~} 2$ (1.0 equiv), $\mathrm{Ar}{ }^{1} \mathrm{~B}(\mathrm{OH})_{2}$ (1.2 equiv), $\mathrm{Pd}\left(\mathrm{PPh}_{3}\right)_{4}(10 \mathrm{~mol} \%), \mathrm{K}_{3} \mathrm{PO}_{4}(2.0$ equiv), toluene- $\mathrm{H}_{2} \mathrm{O}(4: 1), 110^{\circ} \mathrm{C}, 4-6 \mathrm{~h}$.

Table 3 Synthesis of Dissymmetrical 2,5-Diaryl-3,6-dibromothieno[3,2-b]thiophenes $\mathbf{4 a - c}$

\begin{tabular}{llll}
\hline 4 & \multicolumn{2}{c}{$\mathrm{ArB}(\mathrm{OH})_{2}$} & Yield $^{\mathrm{a}}(\%)$ \\
& $\mathrm{Ar}^{1}$ & $\mathrm{Ar}^{2}$ & \\
\hline a & $4-\mathrm{MeC}_{6} \mathrm{H}_{4}$ & $3,5-\mathrm{Me}_{2} \mathrm{C}_{6} \mathrm{H}_{3}$ & 38 \\
b & $3,5-\mathrm{Me}_{2} \mathrm{C}_{6} \mathrm{H}_{3}$ & $\mathrm{Ph}$ & 46 \\
c & $4-t-\mathrm{BuC}_{6} \mathrm{H}_{4}$ & $\mathrm{Ph}$ & 52 \\
\hline
\end{tabular}

${ }^{a}$ Isolated yields.

Further coupling of $\mathbf{3 b}$ or $\mathbf{4 c}$ with 2.4 equivalents of arylboronic acids afforded tetraarylated thieno[3,2-b]thiophenes 5a-c containing four identical aryl groups (Scheme 4 and Table 4). Of note, attempts to prepare triarylated thieno[3,2-b]thiophenes from the dissymmetrical 2,5-diaryl-3,6-dibromothieno[3,2- $b$ ] thiophenes $\mathbf{4 a - c}$ resulted in an inseparable mixture of 2,3,5- and 2,5,6-triarylated thieno[3,2- $b]$ thiophenes.

Interestingly, 1 could also undergo site-selective Heck coupling with 4-methylstyrene, ${ }^{13}$ and Sonogashira reaction with 4-methylphenylethyne (Scheme 5). ${ }^{14}$ 


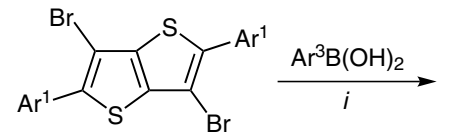

$3 \mathbf{b}$

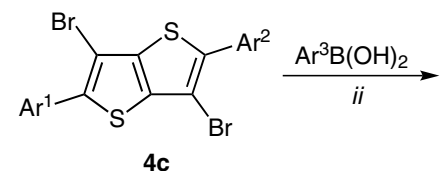<smiles></smiles>

Scheme 4 Synthesis of 5a-c. Reagents and conditions: (i) 5a: 3b (1.0 equiv), $\mathrm{Ar}^{1} \mathrm{~B}(\mathrm{OH})_{2}$ (2.4 equiv), $\mathrm{Pd}\left(\mathrm{PPh}_{3}\right)_{4}(10 \mathrm{~mol} \%), \mathrm{K}_{3} \mathrm{PO}_{4}(4.0$ equiv), toluene- $\mathrm{H}_{2} \mathrm{O}(4: 1), 110{ }^{\circ} \mathrm{C}, 24 \mathrm{~h} ; 5 \mathbf{b}: 3$ (1.0 equiv), $\mathrm{Ar}^{3} \mathrm{~B}(\mathrm{OH})_{2}$ (2.4 equiv), $\mathrm{Pd}\left(\mathrm{PPh}_{3}\right)_{4}(10 \mathrm{~mol} \%), \mathrm{K}_{3} \mathrm{PO}_{4}$ (4.0 equiv), toluene- $\mathrm{H}_{2} \mathrm{O}(4: 1), 110^{\circ} \mathrm{C}, 24 \mathrm{~h}$; (ii) 5c: 4 (1.0 equiv), $\mathrm{Ar}^{3} \mathrm{~B}(\mathrm{OH})_{2}(2.4$ equiv), $\mathrm{Pd}\left(\mathrm{PPh}_{3}\right)_{4}(10 \mathrm{~mol} \%), \mathrm{K}_{3} \mathrm{PO}_{4}$ (4.0 equiv), toluene- $\mathrm{H}_{2} \mathrm{O}(4: 1)$, $110{ }^{\circ} \mathrm{C}, 24 \mathrm{~h}$.

Table 4 Synthesis of Tetraarylthieno[3,2-b]thiophene $\mathbf{5 a - c}$

\begin{tabular}{lllll}
\hline 5 & & $\mathrm{ArB}(\mathrm{OH})_{2}$ & Yield $^{\mathrm{a}}(\%)$ \\
& $\mathrm{Ar}^{1}$ & $\mathrm{Ar}^{2}$ & $\mathrm{Ar}^{3}$ & \\
\hline a & $4-\mathrm{MeC}_{6} \mathrm{H}_{4}$ & - & $4-\mathrm{MeC}_{6} \mathrm{H}_{4}$ & 30 \\
b & $4-\mathrm{MeC}_{6} \mathrm{H}_{4}$ & - & $\mathrm{Ph}$ & 35 \\
c & $4-t-\mathrm{BuC}_{6} \mathrm{H}_{4}$ & $\mathrm{Ph}$ & $4-\mathrm{MeC}_{6} \mathrm{H}_{4}$ & 25 \\
\hline
\end{tabular}

${ }^{\mathrm{a}}$ Isolated yields.
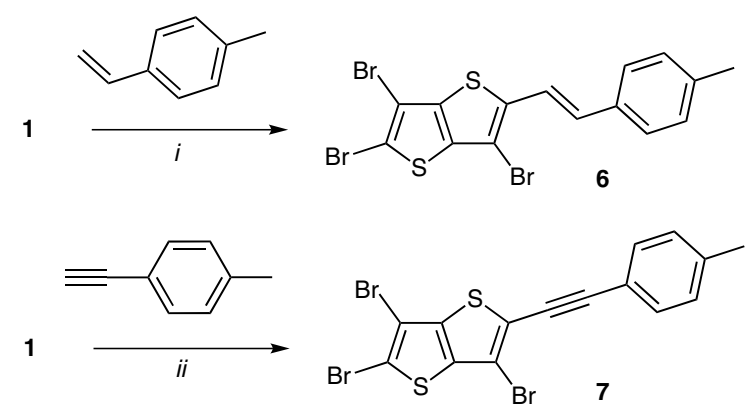

Scheme 5 Synthesis of 6 and 7. Reagents and conditions: (i) 6: 1 (1.0 equiv), 4-methylstyrene (10.0 equiv), $\mathrm{Pd}(\mathrm{OAc})_{2}(10 \mathrm{~mol} \%),(\mathrm{Cy})_{3} \mathrm{P}$ (20 mol\%), DMF, $90{ }^{\circ} \mathrm{C}, 6 \mathrm{~h}$; (ii) 7: 1 (1.0 equiv), arylethyne (1.2 equiv), $\mathrm{Pd}(\mathrm{OAc})_{2}(10 \mathrm{~mol} \%), \mathrm{Ph}_{3} \mathrm{P}(20 \mathrm{~mol} \%)$, $\mathrm{CuI}(20 \mathrm{~mol} \%)$, DMF-Et ${ }_{3} \mathrm{~N}(1: 1), 75^{\circ} \mathrm{C}, 2.5 \mathrm{~h}$.

In conclusion, we have showed that the Suzuki-Miyaura reactions of polybromothieno[3,2-b] thiophene can proceed with predictable site-selectivity, preferably at $\mathrm{C} 2$ and C5. ${ }^{15}$ Controlled synthesis of 2-aryl-3,5,6-tribromothieno[3,2-b] thiophenes, 2,5-diaryl-3,6-dibromothieno[3,2$b]$ thiophenes, and tetraarylated thieno[3,2-b]thiophenes could thus be achieved. Similar regioselectivity was observed for the Heck and Sonogashira couplings of 2,3,5,6tetrabromothieno[3,2-b]thiophenes. Applications of this coupling strategy in the synthesis of materials incorporating thieno[3,2-b]thiophene moieties are now underway in our laboratory, and will be reported in due course.

\section{Acknowledgment}

This research was funded by the Vietnam National Foundation for Science and Technology Development (NAFOSTED) under the grant number 104.01-2012.26.

\section{References and Notes}

(1) Present address: Institut des Sciences Chimiques de Rennes, UMR 6226 CNRS-Université de Rennes 1, Campus de Beaulieu, 35042 Rennes Cedex, France.

(2) (a) Litvinov, V. P.; Goldfarb, Y. A. L. In Advances in Heterocyclic Chemistry; Vol. 19; Katritzky, A. R.; Boulton, A. J., Eds.; Academic Press: San Diego, 1976. (b) Mishra, A.; Ma, C.-Q.; Bäuerle, P. Chem. Rev. 2009, 109, 1141. (c) Liu, Y.; Liu, Q.; Zhang, X.; Ai, L.; Wang, Y.; Peng, R.; Ge, Z. New J. Chem. 2013, 37, 1189. (d) Hergue, N.; Frere, P.; Roncali, J. Org. Biomol. Chem. 2011, 9, 588. (e) Leriche, P.; Raimundo, J.-M.; Turbiez, M.; Monroche, V.; Allain, M.; Sauvage, F.-X.; Roncali, J.; Frere, P.; Skabara, P. J. J. Mater. Chem. 2003, 13, 1324. (f) Ahmed, M. O.; Wang, C.; Keg, P.; Pisula, W.; Lam, Y.-M.; Ong, B. S.; Ng, S.-C.; Chen, Z.-K.; Mhaisalkar, S. G. J. Mater. Chem. 2009, 19, 3449.

(g) Cheng, Y.-J.; Chen, C.-H.; Lin, T.-Y.; Hsu, C.-S. Chem. Asian J. 2012, 7, 818. (h) Burkhardt, S. E.; Conte, S.; Rodriguez-Calero, G. G.; Lowe, M. A.; Qian, H.; Zhou, W.; Gao, J.; Hennig, R. G.; Abruna, H. D. J. Mater. Chem. 2011, 21, 9553. (i) Yamamoto, T.; Nishimura, T.; Mori, T.; Miyazaki, E.; Osaka, I.; Takimiya, K. Org. Lett. 2012, 14, 4914. (j) Yamaguchi, Y.; Maruya, Y.; Katagiri, H.; Nakayama, K.-I.; Ohba, Y. Org. Lett. 2012, 14, 2316. (k) Huang, J.; Luo, H.; Wang, L.; Guo, Y.; Zhang, W.; Chen, H.; Zhu, M.; Liu, Y.; Yu, G. Org. Lett. 2012, 14, 3300. (1) Henson, Z. B.; Müllen, K.; Bazan, G. C. Nature Chem. 2012, 4, 699. (m) Mei, J.; Diao, Y.; Appleton, A. L.; Fang, A. L.; Bao, Z. J. Am. Chem. Soc. 2013, 135, 6724.

(3) Liu, Y.; Sun, X.; Di , C.-A.; Liu, Y.; Du, C.; Lu, K.; Ye, S.; Yu, G. Chem. Asian J. 2010, 5, 1550.

(4) (a) McCulloch, I.; Heeney, M.; Bailey, C.; Genevicius, K.; MacDonald, I.; Shkunov, M.; Sparrowe, D.; Tierney, S.; Wagner, R.; Zhang, W.; Chabinyc, M. L.; Kline, R. J.; McGehee, M. D.; Toney, M. F. Nature Mater. 2006, 5, 328. (b) Niimi, K.; Shinamura, S.; Osaka, I.; Miyazaki, E.; Takimiya, K. J. Am. Chem. Soc. 2011, 133, 8732. (c) Ebata, H.; Izawa, T.; Miyazaki, E.; Takimiya, K.; Ikeda, M.; Kuwabara, H.; Yui, T. J. Am. Chem. Soc. 2007, 129, 15732.

(5) (a) Schröter, S.; Stock, C.; Bach, T. Tetrahedron 2005, 61, 2245. (b) Bellina, F.; Rossi, R. Adv. Synth. Catal. 2010, 352, 8.

(6) (a) Dang, T. T.; Ahmad, R.; Dang, T. T.; Reinke, H.; Langer, P. Tetrahedron Lett. 2008, 49, 1698. (b) Ullah, F.; Dang, T. T.; Heinicke, J.; Villinger, A.; Langer, P. Synlett 2009, 838.

(7) (a) Dang, T. T.; Rasool, N.; Dang, T. T.; Reinke, H.; Langer, P. Tetrahedron Lett. 2007, 48, 845. (b) Tung, D. T.; Tuan, D. T.; Rasool, N.; Villinger, A.; Reinke, H.; Fischer, C.; Langer, P. Adv. Synth. Catal. 2009, 351, 1595. (c) Ehlers, P.; Tung, D. T.; Patonay, T.; Villinger, A.; Langer, P. Eur. J. Org. Chem. 2013, 10, 2000. (d) Tuan, D. T.; Rasool, N.; Tung, D. T.; Reinke, H.; Langer, P. Tetrahedron Lett. 2007, 48,845 .

(8) Tung, D. T.; Villinger, A.; Langer, P. Adv. Synth. Catal. 2008, 350, 2109.

(9) Fuller, S. L.; Iddon, B.; Smith, A. K. J. Chem. Soc., Perkin Trans. 1 1997, 3465.

(10) Hussain, M.; Khera, R. A.; Hung, N. T.; Langer, P. Org. Biomol. Chem. 2011, 9, 370. 
(11) General Procedure for the Synthesis of 2-Aryl-3,5,6tribromothieno[3,2-b]thiophene $2 \mathrm{a}-\mathrm{j}$ : Toluene was degassed by exchanging between vacuum and a stream of argon $(3 \times)$. 2,3,5,6-Tetrabromothieno[3,2-b] thiophene (1.0 equiv) and $\mathrm{Pd}\left(\mathrm{Ph}_{3} \mathrm{P}\right)_{4}(0.05-0.10$ equiv) were dissolved in this degassed toluene $(4 \mathrm{~mL})$ at $60-70^{\circ} \mathrm{C}$. To the obtained solution $\mathrm{H}_{2} \mathrm{O}(1 \mathrm{~mL}), \mathrm{K}_{3} \mathrm{PO}_{4}$ (2.0 equiv), and arylboronic acid (1.2 equiv) were added. The reaction was vigorously stirred under argon atmosphere at $110{ }^{\circ} \mathrm{C}$ until TLC $(100 \%$ hexane) showed the complete consumption of the starting material. The reaction mixture was filtered to remove insoluble particles. The filtrate was washed several times with $\mathrm{H}_{2} \mathrm{O}$, dried over $\mathrm{Na}_{2} \mathrm{SO}_{4}$ and concentrated under reduced pressure by rotary evaporation. The residue was purified by $\mathrm{SiO}_{2}$ column chromatography ( $100 \%$ hexane) to give the product as a white solid. In case of alkoxyphenyl boronic acid, 1,4-dioxane was used instead of toluene (ref. $6 b)$. In fact, toluene- $\mathrm{H}_{2} \mathrm{O}$ gave the same result. 2,3,6Tribromo-5-phenylthieno[3,2-b]thiophene (2a): Starting from $1(230 \mathrm{mg}, 0.5 \mathrm{mmol})$ and phenylboronic acid $(74 \mathrm{mg}$, $0.6 \mathrm{mmol}$ ), 2a was isolated (191 $\mathrm{mg}, 51 \%$ ) as white crystals; $\mathrm{mp} 132-133{ }^{\circ} \mathrm{C} .{ }^{1} \mathrm{H}$ NMR $\left(500 \mathrm{MHz}, \mathrm{CDCl}_{3}\right): \delta=7.68(\mathrm{~m}$, $2 \mathrm{H}, \mathrm{Ar}), 7.45$ (m, $3 \mathrm{H}, \mathrm{Ar}) .{ }^{13} \mathrm{C} \mathrm{NMR}\left(500 \mathrm{MHz}, \mathrm{CDCl}_{3}\right.$ ): $\delta=99.8,106.9,112.5,128.8,128.9,129.0,132.4,136.4$, 139.8. IR (KBr): 3083 (m), 2929 (s), 2905 (m), $1658(\mathrm{~m})$, $1610(\mathrm{~m}), 1582(\mathrm{~m}), 743(\mathrm{~s}), 684(\mathrm{~s}), 588(\mathrm{~m}) \mathrm{cm}^{-1}$. HRMS $(\mathrm{EI}, 70 \mathrm{eV}): \mathrm{m} / z\left(\mathrm{M}^{+},\left[{ }^{79} \mathrm{Br},{ }^{79} \mathrm{Br},{ }^{79} \mathrm{Br}\right]\right)$ calcd for $\mathrm{C}_{12} \mathrm{H}_{5} \mathrm{Br}_{3} \mathrm{~S}_{2}$ : 449.7383; found: 449.7392 .

(12) General Procedure for the Synthesis of 2,5-Diaryl-3,6dibromothieno[3,2-b]thiophenes $4 \mathbf{a}-\mathbf{c}$ : Toluene was degassed by exchanging between vacuum and a stream of argon $(3 \times)$. 2-Ar 1 -3,5,6-tribromothieno[3,2-b]thiophene 2 (1.0 equiv) was dissolved in this degassed toluene $(4 \mathrm{~mL})$ at r.t. To the obtained solution were added $\mathrm{H}_{2} \mathrm{O}(1 \mathrm{~mL}), \mathrm{K}_{3} \mathrm{PO}_{4}$ (2.0 equiv), $\mathrm{Pd}\left(\mathrm{Ph}_{3} \mathrm{P}\right)_{4}\left(0.10\right.$ equiv) and an $\mathrm{Ar}^{2}$ boronic acid (1.2 equiv). The reaction was vigorously stirred under argon atmosphere at $110{ }^{\circ} \mathrm{C}$ until TLC $(100 \%$ hexane) showed the complete consumption of the starting material. The reaction was quenched with $\mathrm{H}_{2} \mathrm{O}$ and the mixture was extracted with EtOAc $(3 \times)$. The extracts were collected, dried over $\mathrm{Na}_{2} \mathrm{SO}_{4}$ and concentrated under reduced pressure by rotary evaporation. The residue was purified by $\mathrm{SiO}_{2}$ column chromatography ( $100 \%$ hexane $\rightarrow 2 \%$ EtOAc in hexane) or by recrystallization from hot toluene to give the product as white crystals.

3,6-Dibromo-2-(3,5-dimethylphenyl)-5-phenylthieno[3,2b]-thiophene (4b): Starting from $2 \mathrm{c}(115 \mathrm{mg}, 0.25 \mathrm{mmol})$ and phenylboronic acid (36.6 $\mathrm{mg}, 0.3 \mathrm{mmol}), 4 \mathbf{b}$ was isolated $(54.7 \mathrm{mg}, 46 \%)$ as a white solid by $\mathrm{SiO}_{2}$ column chromatography ( $100 \%$ hexane); mp $185-186{ }^{\circ} \mathrm{C} .{ }^{1} \mathrm{H}$ NMR $\left(500 \mathrm{MHz}, \mathrm{CDCl}_{3}\right): \delta=7.72(\mathrm{~m}, 2 \mathrm{H}, \mathrm{Ar}), 7.48(\mathrm{~m}, 2 \mathrm{H}, \mathrm{Ar})$, 7.42 (m, $1 \mathrm{H}, \mathrm{Ar}), 7.33$ (s, $2 \mathrm{H}, \mathrm{Ar}), 7.06$ (s, $1 \mathrm{H}, \mathrm{Ar}), 2.40$ (s, $6 \mathrm{H}, 2 \times \mathrm{Me}) .{ }^{13} \mathrm{C} \mathrm{NMR}\left(500 \mathrm{MHz}, \mathrm{CDCl}_{3}\right): \delta=21.3(\mathrm{Me})$, $99.8,100.1,126.8,128.7,128.8,129.1,130.5,132.7,133.0$, 138.4, 138.7, 138.8, 139.2, 139.9. IR (KBr): 3027 (w), 2920 (m), $2870(\mathrm{~m}), 1653(\mathrm{~m}), 1598(\mathrm{~m}), 746(\mathrm{~m}) \mathrm{cm}^{-1}$. HRMS (EI, $\left.70 \mathrm{eV}): \mathrm{m} / z\left(\mathrm{M}^{+},{ }^{79} \mathrm{Br},{ }^{79} \mathrm{Br}\right]\right)$ calcd for $\mathrm{C}_{20} \mathrm{H}_{14} \mathrm{Br}_{2} \mathrm{~S}_{2}: 475.8904$; found: 475.8916 .

(13) Synthesis of 2-(4-Methylstyryl)-3,5,6tribromothieno[3,2-b]thiophene (6): DMF $(4 \mathrm{~mL})$ was saturated with argon by exchanging between vacuum and a stream of $\operatorname{argon}(3 \times) . \mathrm{Pd}(\mathrm{OAc})_{2}(2.8 \mathrm{mg}, 0.0125 \mathrm{mmol}, 0.1$ equiv) and $\mathrm{P}(\mathrm{Cy})_{3}(7.0 \mathrm{mg}, 0.025 \mathrm{mmol}, 0.2$ equiv) were dissolved in this argon-saturated solvent. The brownish yellow solution was stirred at r.t. for further $30 \mathrm{~min}$ to produce the catalyst. 2,3,5,6-Tetrabromothieno[3,2$b]$ thiophene ( $0.57 \mathrm{mg}, 0.125 \mathrm{mmol}, 1.0$ equiv), $\mathrm{Na}_{2} \mathrm{CO}_{3}$ (79.5 mg, $0.75 \mathrm{mmol}, 6.0$ equiv) and 4-methylstyrene ( 295.5 $\mathrm{mg}, 12.5 \mathrm{mmol}, 10.0$ equiv) were added to the solution of the catalyst under a stream of argon. The reaction solution was heated at $90{ }^{\circ} \mathrm{C}$ in $5.5 \mathrm{~h}$ under argon atmosphere. The progress of the reaction was monitored by TLC $(100 \%$ hexane). Besides the monoalkenyl substituted derivative, small amounts of di- and trialkenyl-substituted derivatives were also observed. When the starting material was completely consumed as indicated by TLC, the brownish mixture was allowed to cool to r.t., filtered through Celite to remove the brown precipitate. The filtrate was extracted several times with EtOAc, washed with $\mathrm{H}_{2} \mathrm{O}(3 \times)$ and dried over anhydrous $\mathrm{Na}_{2} \mathrm{SO}_{4}$. The solvent was removed under reduced pressure by rotary evaporation and the residue was purified by $\mathrm{SiO}_{2}$ column chromatography ( $100 \%$ hexane) to give the monoalkenylated 2,3,5,6-tetrabromothieno[3,2b]thiophene as a yellow solid (76.1 mg, 42\%); mp 126-127 ${ }^{\circ} \mathrm{C} .{ }^{1} \mathrm{H}$ NMR $\left(500 \mathrm{MHz}, \mathrm{CDCl}_{3}\right): \delta=7.40(\mathrm{~d}, J=8.0 \mathrm{~Hz}, 2$ $\mathrm{H}, \mathrm{Ar}), 7.20(\mathrm{~d}, J=16.5 \mathrm{~Hz}, 1 \mathrm{H}), 7.17(\mathrm{~d}, J=8.5 \mathrm{~Hz}, 2 \mathrm{H}$, Ar), $6.98(\mathrm{~d}, J=16.0 \mathrm{~Hz}, 1 \mathrm{H}), 2.36(\mathrm{~s}, 3 \mathrm{H}, \mathrm{Me}) .{ }^{13} \mathrm{C} \mathrm{NMR}$ $\left(500 \mathrm{MHz}, \mathrm{CDCl}_{3}\right): \delta=139.4,139.1,138.7,135.1,133.4$, $131.2,129.6,126.7,118.9,112.5,107.2,102.5,21.4$.

(14) General Procedure for the Synthesis of 2-Alkynyl-3,5,6tribromothieno[3,2-b] thiophene 7: A mixture (1:1) of diisopropylamine and THF was saturated with argon by exchanging between vacuum and a stream of argon $(3 \times)$. 2,3,5,6-Tetrabromothieno[3,2- $b]$ thiophene (1.0 equiv), $\mathrm{Pd}(\mathrm{OAc})_{2}\left(0.1\right.$ equiv), $\mathrm{Ph}_{3} \mathrm{P}(0.2$ equiv) and $\mathrm{CuI}(0.2$ equiv $)$ were added to this argon-saturated solution. The suspension was heated to $75^{\circ} \mathrm{C}$ while vigorously stirred until it became homogeneous. To the obtained pale yellow mixture, a solution of arylacetylene (1.2 equiv) in argon-saturated THF $(1.0 \mathrm{~mL})$ was added dropwise in $30 \mathrm{~min}$. The reaction mixture was heated at $75^{\circ} \mathrm{C}$ for $3-6 \mathrm{~h}$. The pale yellow reaction mixture turned reddish brown when the reaction completed as indicated by TLC ( $100 \%$ hexane). The reaction mixture was adsorbed on silica gel, dried under reduced pressure and purified by $\mathrm{SiO}_{2}$ column chromatography to furnish the monoalkynated derivative. Besides the desired product, a significant amount of the symmetric diynes resulting from the homocoupling reaction of the alkynes was separated. All attempts to reduce this by-product were not successful.

2-(4-Methylphenylethynyl)-3,5,6-tribromothieno[3,2b]thiophene (7a): Starting from $1(0.57 \mathrm{mg}, 0.125 \mathrm{mmol})$ and 4-ethynyltoluene $(17.4 \mathrm{mg}, 0.15 \mathrm{mmol}), 7$ was isolated (32 mg, yield 52\%) as a white solid; mp $193-194{ }^{\circ} \mathrm{C} .{ }^{1} \mathrm{H}$ $\operatorname{NMR}\left(500 \mathrm{MHz}, \mathrm{CDCl}_{3}\right): \delta=7.46(\mathrm{~d}, J=8.0 \mathrm{~Hz}, 2 \mathrm{H}, \mathrm{Ar})$, $7.18(\mathrm{~d}, J=8.0 \mathrm{~Hz}, 2 \mathrm{H}, \mathrm{Ar}), 2.38(\mathrm{~s}, 3 \mathrm{H}, \mathrm{Me}) .{ }^{13} \mathrm{C} \mathrm{NMR}$ $\left(500 \mathrm{MHz}, \mathrm{CDCl}_{3}\right): \delta=139.6,138.0,131.7,131.5,129.3$, 119.0, 114.8, 107.9, 107.1, 99.9, 80.5, 21.6.

(15) CCDC 971825 contains the supplementary crystallographic data for this paper. These data can be obtained free of charge from The Cambridge Crystallographic Data Centre via www.ccdc.cam.ac.uk/data_request/cif. 